\title{
Étude de cas
}

\section{Mise en évidence d'une soumission chimique à la niaprazine chez des enfants par analyse des cheveux en CL-SM/SM}

\section{Determination of niaprazine-facilitated sedation in children by hair analysis with LC-MS/MS}

\author{
Marion Villain ${ }^{\star}$, Emilie Vallet, Vincent Cirimele, Pascal Kintz \\ Laboratoire ChemTox, 67400 Illkirch, France
}

\begin{abstract}
Résumé - La niaprazine, commercialisée sous la dénomination de Nopron ${ }^{\circledR}$, est largement utilisée en France pour ses effets hypnotiques dans le cas des insomnies occasionnelles chez l'enfant. Ce produit est accessible sans ordonnance médicale. Le premier cas de la littérature impliquant l'administration répétitive de niaprazine comme arme chimique dans le but d'une agression sexuelle est présenté ci-dessous. Trois enfants ( 2 filles et 1 garçon) ont été sédatés et agressés par leur beau-père de façon répétée entre 2002 et 2006. Selon la demande du juge en charge de cette affaire, les cheveux des trois enfants ont été prélevés afin de réaliser une analyse segmentaire dans le but d'y rechercher des sédatifs (30 molécules) par chromatographie liquide couplée à la spectrométrie de masse en tandem. De la niaprazine a été retrouvé dans les cheveux des trois enfants à des concentrations allant de 21 à $3431 \mathrm{pg} / \mathrm{mg}$. Ces concentrations, démontrant l'absorption répétée de niaprazine, ne peuvent pas être comparées à des résultats antérieurs, par manque de référence sur le sujet.
\end{abstract}

Mots clés : Niaprazine, enfants, soumission chimique, CL-SM/SM

\begin{abstract}
Niaprazine, under the trade name Nopron, is largely used in France as an hypnotic agent for occasional insomnia of children. This compound is available without medical prescription. The first case involving repetitive administration of niaprazine as a drug-facilitated sexual assault is reported. Three children ( 2 girls and 1 boy) were repetitively sedated and assaulted by their father-in-law between 2002 and 2006. Niaprazine's liquid formulation represents a good potential access to surreptitiously administer it in beverages. According to the request of the judge in charge of this case, hairs of victims were collected, segmented and screened for sedatives by LC-MS/MS. Niaprazine was detected in the range 21 to $3431 \mathrm{pg} / \mathrm{mg}$ for the three children. These concentrations could not be compared with previous results, due to a lack of literature.
\end{abstract}

Key words: Niaprazine, children, drug-facilitated sexual assault, LC-MS/MS

Reçu le 10 juillet 2008, accepté après modifications le 22 septembre 2008

Publication en ligne le 31 janvier 2009

\section{Introduction}

La soumission chimique peut être définie comme l'administration de substances psycho-actives à une personne à des fins délictueuses ou criminelles. Même si la majorité des observations concernent des filles jeunes à qui leur agresseur administre une substance afin de diminuer leur résistance à l'acte sexuel [1], les enfants [2,3] et les personnes âgées [4] apparaissent comme des victimes potentielles. Dans ces cas, l'empoisonnement par un membre de la famille est fréquent : enfants chimiquement battus pour obtenir une sédation à visée pédophile et personnes âgés sédatées pour détourner leur vigi-

${ }^{\star}$ Correspondance : Marion Villain, Tel : +33 (0)390 4005 40, Fax : +33 (0)390 4005 41, mvillain@ labochemtox.com lance (escroquerie au chéquier ou à la carte bleue). Le but peut aussi être tout simplement d' « avoir la paix ».

Les produits sont majoritairement incorporés dans des boissons. Selon les agresseurs, le produit idéal est celui qui est actif à faible dose, rapidement soluble en milieu aqueux, sans goût, et dont les effets sont rapides à s'installer, tout en provoquant une amnésie des faits. Les produits les plus utilisés sont les suivants : alcool éthylique, benzodiazépines, hypnotiques, anesthésiques, hallucinogènes et médicaments détournés de leur AMM comme la niaprazine. La forme sirop de cette dernière est favorable à une introduction discrète dans les liquides.

Le sang et les urines sont les matrices conventionnelles pour documenter les cas de soumission chimique. Cependant, 
l'analyse des cheveux est complémentaire et nécessaire puisqu'elle permet de tracer l'historique de consommation et de différencier exposition unique et répétée à un xénobiotique, par simple segmentation de la mèche de cheveux $[5,6]$.

Nous présentons ici la méthode de dosage de la niaprazine dans les cheveux par CL-SM/SM et son application dans un cas médico-légal impliquant trois enfants.

\section{Matériels et méthodes}

\section{1 Échantillons}

Les mèches de cheveux appartenant aux trois enfants concernés ont été prélevées par un médecin. Ces enfants étaient sédatés et agressés par leur beau-père de façon répétée entre 2002 et 2006. La juge en charge de cette affaire nous a fait parvenir les mèches afin de réaliser une analyse segmentaire dans le but d'y rechercher des sédatifs (30 molécules) par chromatographie liquide couplée à la spectrométrie de masse en tandem. Les cheveux des deux filles, d'une longueur supérieure à $10 \mathrm{~cm}$, ont été coupés en 3 segments de $3 \mathrm{~cm}$ alors que la mèche de cheveux du garçon, de $5 \mathrm{~cm}$ de long, a été coupée en deux segments de $2,5 \mathrm{~cm}$.

Les analyses de ces différentes sections ont ainsi permis de retracer l'historique de consommation sur environ 9 mois avant le prélèvement pour les filles et 5 mois pour le garçon. Il est en effet admis par la communauté scientifique que les cheveux poussent d'environ $1 \mathrm{~cm}$ par mois.

\subsection{Extraction}

La méthode d'analyse est une extension de celle publiée précédemment [7].

Après décontamination de chaque mèche de cheveux dans 2 bains de dichlorométhane, les segments ont été finement coupés aux ciseaux $(<1 \mathrm{~mm})$. L'incubation de $20 \mathrm{mg}$ a été réalisée dans $1 \mathrm{~mL}$ de tampon phosphate $\mathrm{pH} 8,4$ pendant la nuit. La phase aqueuse a été extraite par $5 \mathrm{~mL}$ d'un mélange de dichlorométhane/éther (80/20), en présence de $1 \mathrm{ng}$ de diazepam-d $\mathrm{d}_{5}$ utilisé comme étalon interne (EI). Après reprise de l'extrait sec dans $100 \mu \mathrm{L}$ d'un mélange acétonitrile/tampon formiate $2 \mathrm{mM}+0.1 \%$ d'acide formique (5/95), $15 \mu \mathrm{L}$ ont été injectés sur une colonne XTerra MS C18 $(100 \times 2.1 \mathrm{~mm}, 3.5 \mu \mathrm{m})$ d'un système Accela Thermo Fischer Scientific (Waltham, MA, USA).

\subsection{Procédure CL-SM/SM}

La chromatographie s'est déroulée avec un gradient d'acétonitrile et de tampon formiate à un débit de $0.2 \mathrm{~mL} / \mathrm{min}(5 \%$ acetonitrile - $95 \%$ tampon formiate avec $0.1 \%$ d'acide formique jusqu'à $80-20 \%$ à 9 min). Le détecteur de masse était un triple-quadripole Quantum Ultra (Thermo Fischer Scientific) fonctionnant en électrospray dans le mode ionisation positive $(\mathrm{ES}+)$. Le temps total d'analyse était de $16 \mathrm{~min}$.

Les conditions suivantes ont été optimisées pour la niaprazine ainsi que pour les 29 autres molécules faisant partie du
Tableau I. Transitions et énergies de collision optimisées pour la niaprazine et pour l'EI.

\begin{tabular}{cccc}
\hline Composé & $m / z$ parent & $m / z$ fils & $\begin{array}{c}\text { Énergie de } \\
\text { collision }\end{array}$ \\
\hline Niaprazine & 357 & 106177 & 3621 \\
\hline Diazepam-d $_{\mathbf{5}}(\mathrm{EI})$ & 290 & 154198 & 2734 \\
\hline
\end{tabular}

Tableau II. Concentrations en niaprazine retrouvées dans les différents segments de cheveux des trois enfants.

\begin{tabular}{ccc}
\hline Enfant & Segment & Niaprazine $(\mathrm{pg} / \mathrm{mg})$ \\
\hline Fille 1 & $0-3 \mathrm{~cm}$ & 382 \\
& $3-6 \mathrm{~cm}$ & 21 \\
& $6-9 \mathrm{~cm}$ & 21 \\
\hline Fille 2 & $0-3 \mathrm{~cm}$ & 315 \\
& $3-6 \mathrm{~cm}$ & $<10$ \\
& $6-9 \mathrm{~cm}$ & $<10$ \\
\hline Garçon & $0-2,5 \mathrm{~cm}$ & 3431 \\
& $2,5-5 \mathrm{~cm}$ & 2642 \\
\hline
\end{tabular}

« screening sédatifs ». Un spray voltage de $4000 \mathrm{~V}$, une température de capillaire de $350{ }^{\circ} \mathrm{C}$, des gaz de nébulisation et de désolvatation à 20 et 30 (unités arbitraires) et une pression dans la cellule de collision à 1.5 mTorr d'argon. Pour la niaprazine, l'offset à été optimisé à $5 \mathrm{~V}$ et le tube lens à $100 \mathrm{~V}$.

Toutes les molécules ont été identifiées par 2 transitions; celles de la niaprazine, de l'étalon interne ainsi que les différents critères d'optimisation sont rapportés dans le tableau I. La limite de quantification de la niaprazine a été fixée à $10 \mathrm{pg} / \mathrm{mg}$.

\subsection{Résultats et discussion}

Les concentrations retrouvées dans les différents segments sont rapportées dans le tableau II. Les concentrations de niaprazine démontrent une exposition à ce produit sans qu'il soit possible d'en déterminer le nombre, par manque d'étude contrôlée. Le chromatogramme d'un segment positif est présenté en figure 1 . La fille 2, pour laquelle la niaprazine a été identifiée uniquement dans le premier segment $(3 \mathrm{~cm}$, $315 \mathrm{pg} / \mathrm{mg}$ ), a été exposée de façon répétée à ce produit durant les trois mois précédant le prélèvement. Le fait d'identifier la molécule dans différents segments pour la fille $1(3 \times 3 \mathrm{~cm}$, $382,21$ et $21 \mathrm{pg} / \mathrm{mg})$ et pour le garçon $(2 \times 2,5 \mathrm{~cm}, 3431$ et $2642 \mathrm{pg} / \mathrm{mg}$ ) démontre que les expositions ont eu lieu sur une période plus longue. En effet, les trois segments positifs de la fille 1, d'une longueur totale de $9 \mathrm{~cm}$, objectivent une exposition continue sur les 9 mois précédant le prélèvement (de façon plus occasionnelle sur les périodes les plus anciennes). Pour le garçon, l'exposition apparaît massive et répétée sur une période de 5 mois. Chez les trois enfants, le segment proximal (correspondant à la période la plus récente), est le plus concentré, indiquant une exposition plus importante dans les derniers mois. Enfin, compte tenu des concentrations, il a été possible de conclure que le garçon avait été le plus exposé. 


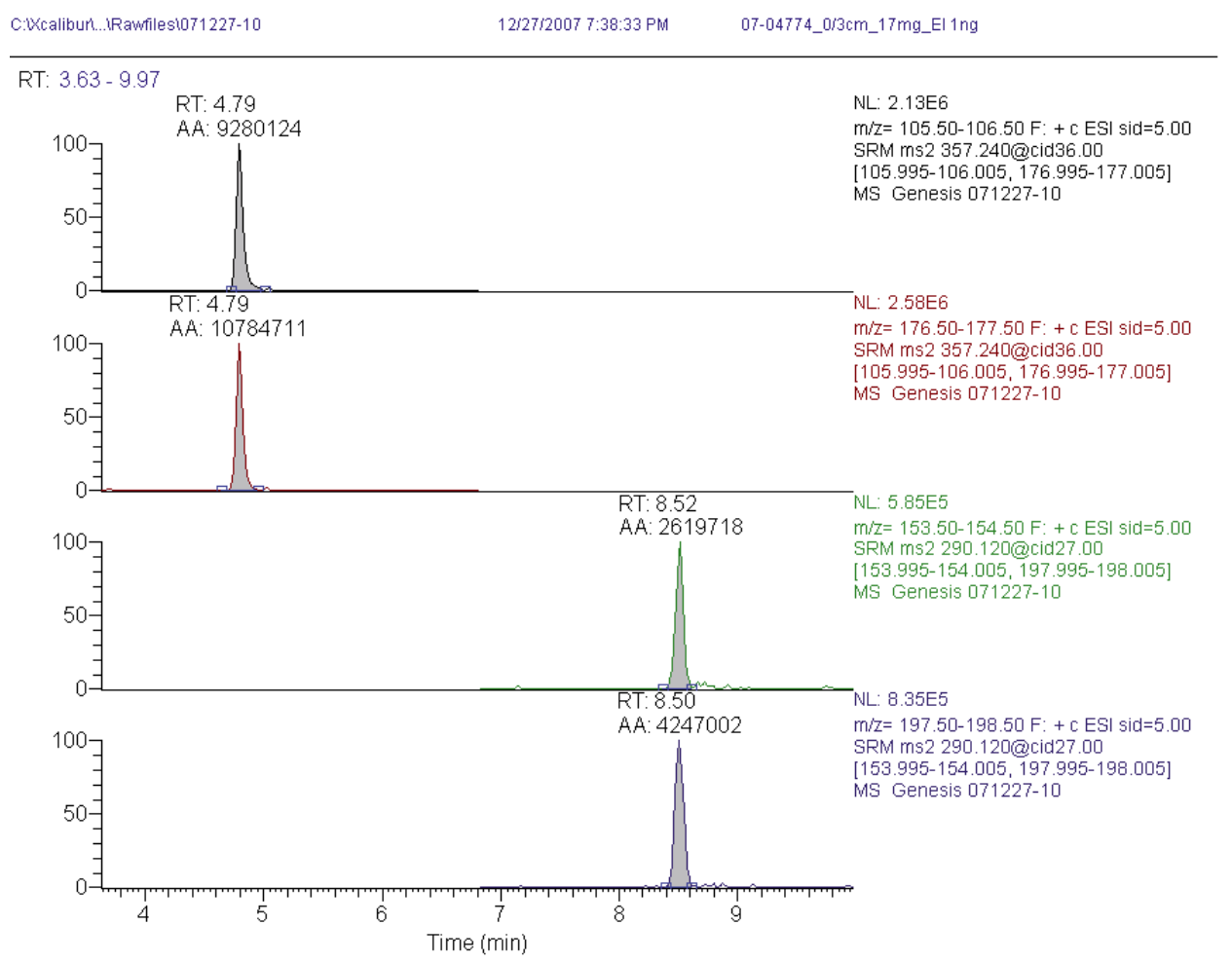

Fig. 1. Signal chromatographique de la niaprazine (en haut) retrouvée dans le segment proximal de la fille 1. La concentration, calculée par rapport au diazepam- $\mathrm{d}_{5}$ (en bas) est mesurée à $382 \mathrm{pg} / \mathrm{mg}$.

\section{Conclusion}

L'analyse des cheveux est indispensable pour retracer un historique de consommation, nécessaire dans les affaires d'empoisonnement d'enfants. L'administration de niaprazine dans le but d'obtenir une sédation est considérée comme de la soumission chimique, même et surtout dans l'enceinte familiale. La forme liquide de la niaprazine en fait un produit de choix dans ce genre de situations. Selon la loi française, cette molécule peut être considérée comme une arme chimique.

\section{Références}

1. Bechtel LK, Holstege CP. Criminal poisoning: Drug facilitated sexual assault. Emerg Med Clin North Am. 2007; 25: 499-525.

2. Gaillard Y, Masson-Seyer MF, Giroud M, Roussot JF, Prevosto JM. A case of drug-facilitated sexual assault leading to death by chloroform poisoning. Int J Legal Med. 2005; 30: 1-5.
3. Kintz P, Villain M, Evans J, Pujol ML, Salquebre G, Cirimele V. A case of abuse in which children were forced to take tablets containing scopolamine: segmental analysis of hair for scopolamine by ultra performance liquid chromatography - mass spectrometry. Forensic Toxicol. 2007; 25: 49-52.

4. Kintz P, Villain M, Cirimele V. Chemical abuse in the elderly: evidence from hair analysis. Ther Drug Monit. 2008; 30: 218-224.

5. Chèze M, Duffort G, Deveaux M, Pépin G. Hair analysis by liquid chromatography-tandem mass spectrometry in toxicological investigation of drug-facilitated crimes: report of 128 cases over the period June 2003 - May 2004 in metropolitan Paris. Forensic Sci Int. 2005; 153: 3-10.

6. Villain M, Chèze M, Tracqui A, Ludes B, Kintz P. Windows of detection of zolpidem in urine and hair. Application to two drugfacilitated sexual assaults. Forensic Sci Int. 2004; 143: 157-161.

7. Villain M, Concheiro M, Cirimele V, Ludes B, Kintz P. Screening method for benzodiazepines and hypnotics in hair at $\mathrm{pg} / \mathrm{mg}$ level by LC-MS/MS. J Chromatogr B. 2005; 825: 72-78. 